\title{
Cryogenically Cooled Ultra Low Vibration Silicon Mirrors for Gravitational Wave Observatories
}

\author{
Brett Shapiro $^{1, *}$, Rana X Adhikari², Odylio Aguiar ${ }^{3}$, Edgard \\ Bonilla ${ }^{1}$, Danyang Fan ${ }^{1}$, Litawn Gan ${ }^{1}$, Ian Gomez ${ }^{1}$, Sanditi \\ Khandelwal $^{1}$, Brian Lantz ${ }^{1}$, Tim MacDonald ${ }^{1}$, Dakota \\ Madden-Fong ${ }^{4}$ \\ ${ }^{1}$ E.L. Ginzton Laboratory, Stanford University, Stanford, CA 94305 \\ ${ }^{2}$ LIGO Laboratory, California Institute of Technology, 1200 East California \\ Boulevard, Pasadena, CA 91125 \\ ${ }^{3}$ Instituto Nacional de Pesquisas Espaciais (INPE), Astrophysics Division, Avenida \\ dos Astronautas 1758, 12227-010 - São José dos Campos, SP, Brazil \\ ${ }^{4}$ Willamette University, 900 State Street, Salem, Oregon 97301 \\ * Corresponding author. E-mail: shapirob@stanford.edu
}

\begin{abstract}
Interferometric gravitational wave observatories recently launched a new field of gravitational wave astronomy with the first detections of gravitational waves in 2015. The number and quality of these detections is limited in part by thermally induced vibrations in the mirrors, which show up as noise in these interferometers. One way to reduce this thermally induced noise is to use low temperature mirrors made of high purity single-crystalline silicon. However, these low temperatures must be achieved without increasing the mechanical vibration of the mirror surface or the vibration of any surface within close proximity to the mirrors. The vibration of either surface can impose a noise inducing phase shift on the light within the interferometer or physically push the mirror through oscillating radiation pressure. This paper proposes a system for the Laser Interferometric Gravitational-wave Observatory (LIGO) to achieve the dual goals of low temperature and low vibration to reduce the thermally induced noise in silicon mirrors. Experimental results are obtained at Stanford University to prove that these dual goals can be realized simultaneously.
\end{abstract}

Keywords: low vibration cryogenics, gravitational waves, feedback control

(C) 2016. This manuscript version is made available under the Elsevier user license http://www.elsevier.com/open-access/userlicense/1.0/ 


\section{Introduction}

Gravitational waves were directly observed for the first time [1, 2] by the Advanced Laser Interferometer Gravitational-wave Observatory (LIGO) [3, 4] in 2015, 100 years after Einstein first described them [5]. Advanced LIGO is expected to be sensitive to waves coming from binary black hole inspirals, binary neutron star inspirals, stellar collapses, pulsars, and a stochastic background of radiation from the early universe. So far, the two confirmed detections made by Advanced LIGO in its first four month observing run were from binary black hole inspirals.

Advanced LIGO's observations are limited in part by the displacement noise of the mirrors in the interferometer. While Advanced LIGO is expected to continue making significant observations of astrophysical events, more advanced observatories will nonetheless be required in order to realize the potential of the astronomy and astrophysics obtainable with gravitational waves.

The lowest noise frequency band of Advanced LIGO is designed to be limited by thermally driven vibrations of the $40 \mathrm{~kg}, 34 \mathrm{~cm}$ diameter, room temperature, fused silica mirrors. This noise can be significantly reduced through a combination of lowering the mirrors' temperatures to a cryogenic regime, selecting mirror materials with low mechanical loss, and using larger mirror diameters. The next generation of LIGO, LIGO Voyager, plans to upgrade the existing facilities, in part, with a switch to $44 \mathrm{~cm}$ diameter, $124 \mathrm{~K}$, silicon mirrors between $150 \mathrm{~kg}$ and $200 \mathrm{~kg}$. Silicon is beneficial at cryogenic temperatures because unlike fused silica, its mechanical loss decreases with temperature. $124 \mathrm{~K}$ is chosen because silicon's thermal expansion coefficient crosses zero [6]. This zero crossing eliminates the thermoelastic component of the thermal noise, and also minimizes radius of curvature changes induced by temperature gradients. Also unlike fused silica, cryogenic silicon has very high thermal conductivity. Higher thermal conductivity permits higher laser powers because the thermal lensing of the mirror is reduced, and because getting absorbed heat out is easier. High laser power reduces high frequency quantum noise (shot noise). LIGO Voyager will have about $3 \mathrm{MW}$ of power on the mirror, while Advanced LIGO will have about $750 \mathrm{~kW}$. Because of these features of silicon at $124 \mathrm{~K}$, the LIGO Voyager noise will be about 3 times smaller than Advanced LIGO over a wide frequency band.

Fig. 1 shows a prediction for the displacement noise performance for a possible LIGO Voyager interferometer in black. For reference, the Advanced LIGO design is shown as the dashed cyan curve. The other curves show the contributions to the total LIGO Voyager noise. The mirror thermal noise, in yellow, is achieved by utilizing $124 \mathrm{~K}$ silicon mirrors. The precise values of the LIGO Voyager curves will depend somewhat on design choices still to be made, but these curves are a good representation for the work presented here.

The mirror's low temperature must be achieved without increasing its mechanical vibration, i.e. without increasing the blue curve in Fig. 1. Additionally, any cryogenic components near the mirror must also have low mechanical vibration due to noise 
coupling through scattered laser light. Small components of the interferometer laser will scatter off the mirrors, hit nearby surfaces, and then recombine back into the interferometer. If these surfaces are vibrating they will impose both a phase shift on the light, and also cause disturbances in the light's radiation pressure on the mirror. Both effects will cause increased noise to appear in the interferometer.

Other future gravitational wave observatories such as KAGRA in Japan [7] and The Einstein Telescope (ET) in Europe [8] plan to use cryogenic mirrors as well, but at the colder temperatures of $20 \mathrm{~K}$ and $10 \mathrm{~K}$ respectively. ET, uniquely, plans to use both $10 \mathrm{~K}$ and room temperature mirrors. The different temperature regimes emphasize different frequency bands of performance and different engineering challenges. The lower temperatures help further reduce thermal noise, visible below about $100 \mathrm{~Hz}$. However, high laser power on the mirror to help reduce shot noise above $100 \mathrm{~Hz}$ is not feasible at these temperatures. Lower temperatures do permit smaller mirrors, since a large laser radius is not needed to average out thermally induced oscillations seen in the mirror coating and since the laser's radiation pressure noise will be weaker (low frequency quantum noise). Cooling the mirrors this low does require building a conductive heat path into the mirror's vibration isolation system, which imposes a significant design challenge, as the heat path can compromise the vibration.

The $3 \mathrm{MW}$ of power LIGO Voyager intends to use is much greater than the $400 \mathrm{~kW}$ for KAGRA's and $18 \mathrm{~kW}$ for ET's cryogenic mirrors. This higher laser power permits lower shot noise. Additionally, the warmer cryogenic temperatures in LIGO Voyager permit radiative cooling of the mirrors, so a conductive heat path does not need to be integrated into the vibration isolation system. Larger diameter and more massive mirrors are needed, however, to help minimize the effect of mirror thermal noise and radiation pressure. The high laser power does increase the potential for light scattering noise, so engineering work is required to keep this under control.

The contribution of this paper is to propose for the first time a sufficiently low vibration cryogenic system for the $124 \mathrm{~K}$ silicon mirrors intended for future LIGO Voyager interferometers. Section 2 describes the proposed cryogenic layout. Section 3 describes the experimental setup at Stanford, used to demonstrate that sufficiently low levels of vibration are feasible. Section 4 presents and analyses the results from this experiment. Finally, Section 5 presents concluding remarks.

\section{Proposed LIGO Voyager Cryogenic Layout}

\subsection{Overview}

Fig. 2 illustrates the layout of a LIGO mirror vacuum chamber with the proposed cryogenic infrastructure. The vacuum enclosure and the vibration isolation table will be reused from Advanced LIGO. The layout of the cryogenic components is intended to be general in scope. Details such as the precise dimensions of the shields and the materials used will be determined in future work. 


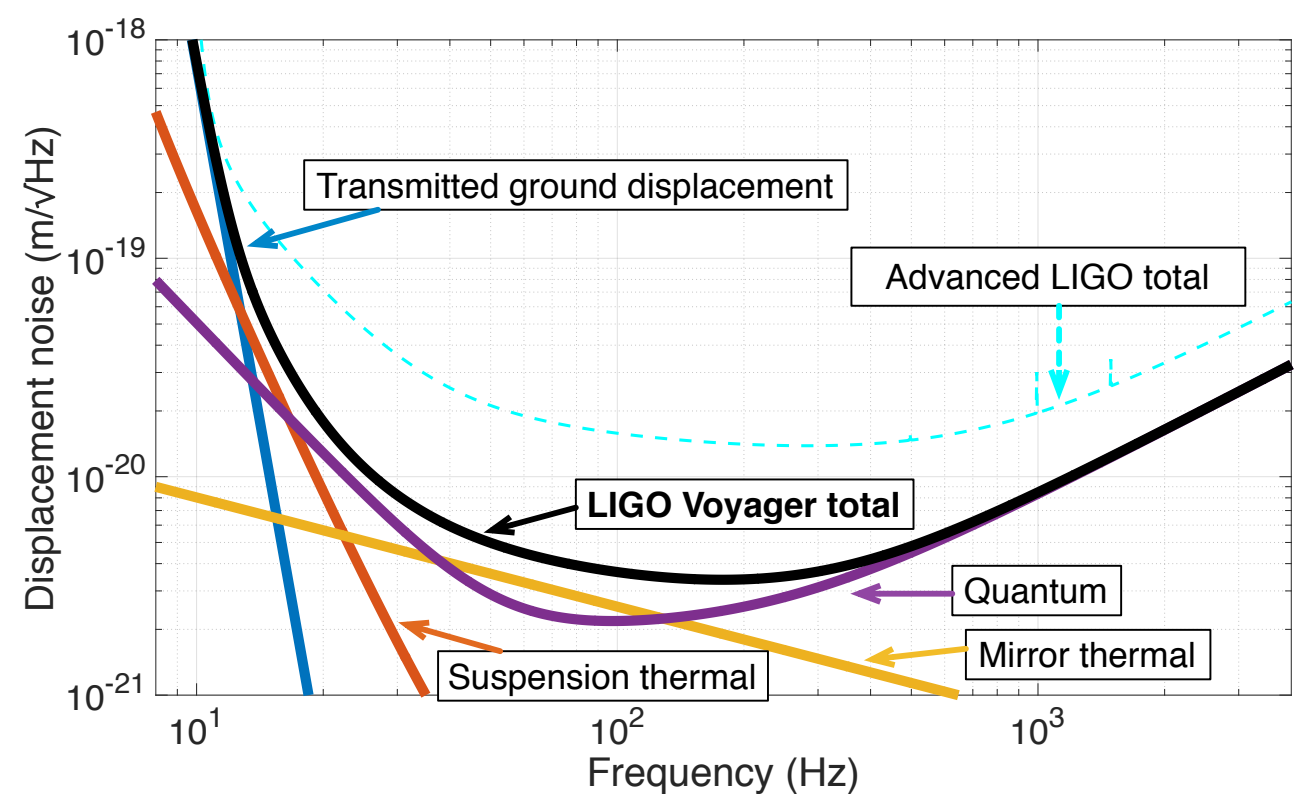

Figure 1. The primary contributing noise sources for a possible LIGO Voyager interferometer design. Black, the predicted total displacement noise. Yellow, the thermal noise of the mirror surface. Red, the thermal noise associated with the suspension elements supporting the mirror. Blue, the transmission of ground displacements. Purple, quantum noise: high frequencies are shot noise, low frequencies are radiation pressure noise from photons bouncing off the mirror surfaces. Dashed cyan, a reference curve of the Advanced LIGO design noise.

The mirrors are between $150 \mathrm{~kg}$ and $200 \mathrm{~kg}$ of $124 \mathrm{~K}$ silicon. To provide the necessary vibration isolation, the mirrors are the bottom stage of a four stage suspension, which hangs from an actively controlled vibration isolation table. The isolation table is a two stage mass-spring system that feeds back on-board inertial sensor signals to actuators in order to achieve favorable isolation performance below $10 \mathrm{~Hz}$. The four stage suspension is a passive isolation system providing isolation above $10 \mathrm{~Hz}$.

To achieve the desired mirror temperature without compromising this vibration isolation system, the cryogenic system and vibration systems are physically separate. As shown in Fig. 2, the cryogenic system consists of a dual heat shield system that surrounds the lower part of the suspension with a non-contacting inner shield at $83 \mathrm{~K}$ and an outer shield at $80 \mathrm{~K}$. The inner shield maintains the mirror temperature purely by radiatively absorbing about $5 \mathrm{~W} .5 \mathrm{~W}$ is the expected steady state heat load on the mirror, primarily due to absorption of the interferometer laser into the mirror coating. Conductive heat transfer through the mirror's supporting wires is negligible given the wires' long length and small cross-section. Although the inner shield is not in direct contact with any part of the vibration isolation system, it must also be well isolated, though not to the same extent as the mirror itself. Its isolation requirement is dominated by noise couplings resulting from scattered laser light. 


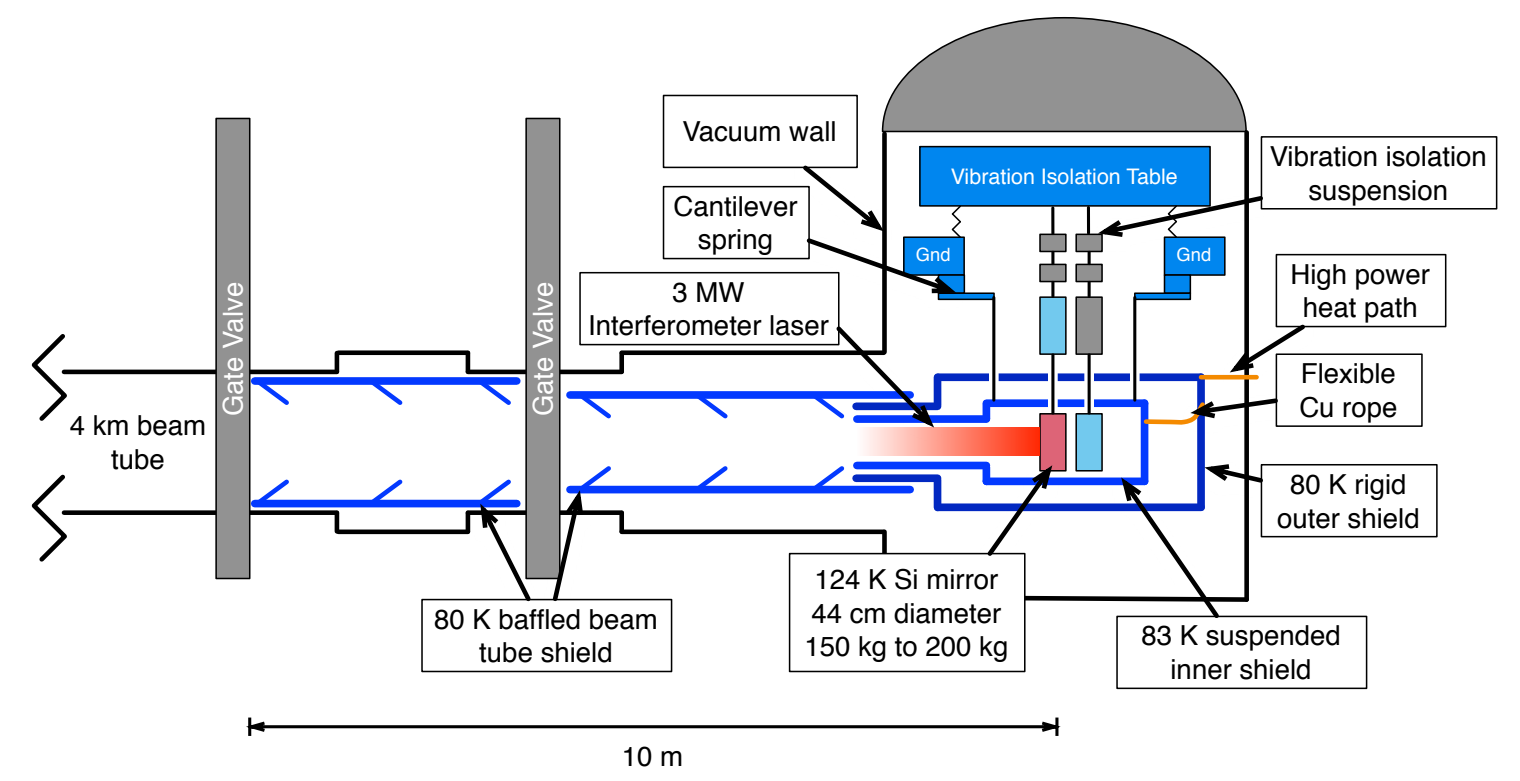

Figure 2. A sketch of a cryogenic mirror and the supporting vibration isolation system within the LIGO vacuum enclosure. The inner, outer, and beam tube shields are cryogenic, along with any components they enclose. All other components are room temperature. The inner shield sensors and actuators are not shown here.

In order to reach its vibration requirement, the inner shield is suspended from the ground and actively controlled to follow the position of the well isolated vibration isolation table. In this way, the shield can achieve low frequency isolation similar to that of the vibration isolation table without the need to mount any cryogenics on the table itself. The inner shield is then cooled conductively from the outer shield by copper ropes flexible enough to not compromise the inner shield's vibration isolation.

The flexibility of these copper ropes limits their heat transfer capabilities to about $100 \mathrm{~W}$. These $100 \mathrm{~W}$ consist of about $5 \mathrm{~W}$ of heat from the mirror, $50 \mathrm{~W}$ from scattered laser light, and the remainder from the conductive heat load from the surrounding environment. Therefore, the outer shield's primary role is to limit the heat load on the inner shield by shielding it from radiation emitted by the surrounding room temperature environment. Since scattered interferometer light cannot reach the outer shield, the outer shield's isolation requirement is relaxed enough that it does not need isolation from the ground. Consequently, it can be cooled directly with liquid nitrogen pipes or thick heat links.

In order to get the interferometer's laser beam through both heat shields without causing a large heat leak, a shield is used inside the laser's beam tube. This beam tube shield extends through the first $10 \mathrm{~m}$ of the $4 \mathrm{~km}$ long beam tube and has an inner diameter greater than or equal to that of the mirror, about $44 \mathrm{~cm}$. At this diameter, $10 \mathrm{~m}$ is long enough to reduce the heat leak through the hole to $0.5 \mathrm{~W}, 10 \%$ of the laser heat load [9]. Conical baffles, shown by the slanted lines inside the shield in Fig. 2, block small angle specular reflection from funneling warm light towards the mirror. 
Measurements done at the KAGRA interferometer under construction in Japan show that these baffles improve the shields performance by a factor of 1000 [10, 11]. The beam tube shield is suspended from the beam tube to provide a nominal amount of vibration isolation, but it is not actively controlled. Being further from the mirror, its vibration isolation requirements are not as strict as the inner shield.

For each shield, all surfaces except those exposed directly to the warm environment are coated black to improve the absorption of heat radiation. Radiative heat transport is particularly important for the mirror, since this is the only mechanism for maintaining the mirror's steady state temperature. The cylindrical barrel of the mirror is also coated black. The shield surfaces directly exposed to the environment have multiple layers of low emissivity shielding to reduce their ambient heat load.

\subsection{Initial cool-down}

While radiative heat transfer alone is sufficient to maintain the mirror at $124 \mathrm{~K}$, it is not sufficient for reaching that temperature in a reasonable amount of time. It can be estimated that with radiative cooling alone, a $200 \mathrm{~kg}$ silicon mirror will take about 1 month to reach this temperature from room temperature. Cooling times on the order of days are more desirable for maximizing the duty cycle of a cryogenic observatory.

To achieve faster cooling rates, a movable heat link (not shown in Fig. 2) will come into contact with the mirror during the cool-down period. It will then move away during the operation of the interferometer. The thermal conductivity of this contact will be enhanced through the application of mechanical pressure and/or exchange gas.

\subsection{Shield vibration isolation}

As mentioned previously, the inner shield requires isolation from ground displacements to minimize noise couplings to the interferometer through scattered laser light. This light scatters from the mirror, to the inner shield, and then back to the mirror. Along the way, the light picks up signals from the shield's vibration, which are then deposited into the interferometer as amplitude and phase noise. This noise is minimized by coating the inside of the shield black and by minimizing its displacement. Even with typical black coatings, the shield's displacement must be pushed below that of the ambient ground displacements.

Another noise source occurs from the gravitational force exerted between the shields and the mirror. This noise exists for both the inner and outer shields. The force changes as their relative positions to the mirror change in time through gravitational gradients. While not shown here, for expected shield mass values and geometries, this gravitational noise is small enough to neglect. The derivations of the scattered light noise and gravitational noises are beyond the scope of this paper and will be presented in a forthcoming publication. 
2.3.1. Inner shield displacement limit The dashed black curve in Fig. 3 shows the maximum permitted displacement spectrum for the inner shield in order to achieve negligible scattered light noise in the interferometer. Since the exact dimensions of the shield are not yet known, this curve is for an idealized spherical shield. The scattered light noise is inversely proportional to the shield radius. Larger shields can tolerate larger displacements. This curve assumes a $0.2 \mathrm{~m}$ radius, due to space constraints.

This limit curve assumes a scatter profile for the mirror (BRDF) similar to that of Advanced LIGO. The heat shield is assumed to be coated with Vantablack, which has a BRDF of 0.01, constant over the incident angle of the light [12]. The laser power on the mirror is $3 \mathrm{MW}$. Because of this high power, the scattered light noise is dominated by radiation pressure fluctuations on the mirror up to $40 \mathrm{~Hz}$. Above $40 \mathrm{~Hz}$, the noise is from phase fluctuations in the light.

In addition to the inner shield displacement limit, Fig. 3 shows measured ground motion in red and yellow at the LIGO Livingston Observatory (LLO). The two measurements are a near worst case. They represent the 95th percentile ground displacement during August and December of 2009. The 95th percentile indicates that the ground displacement was less than this $95 \%$ of the time [13]. The inner shield will require enough vibration isolation to suppress these curves below the dashed limit curve.

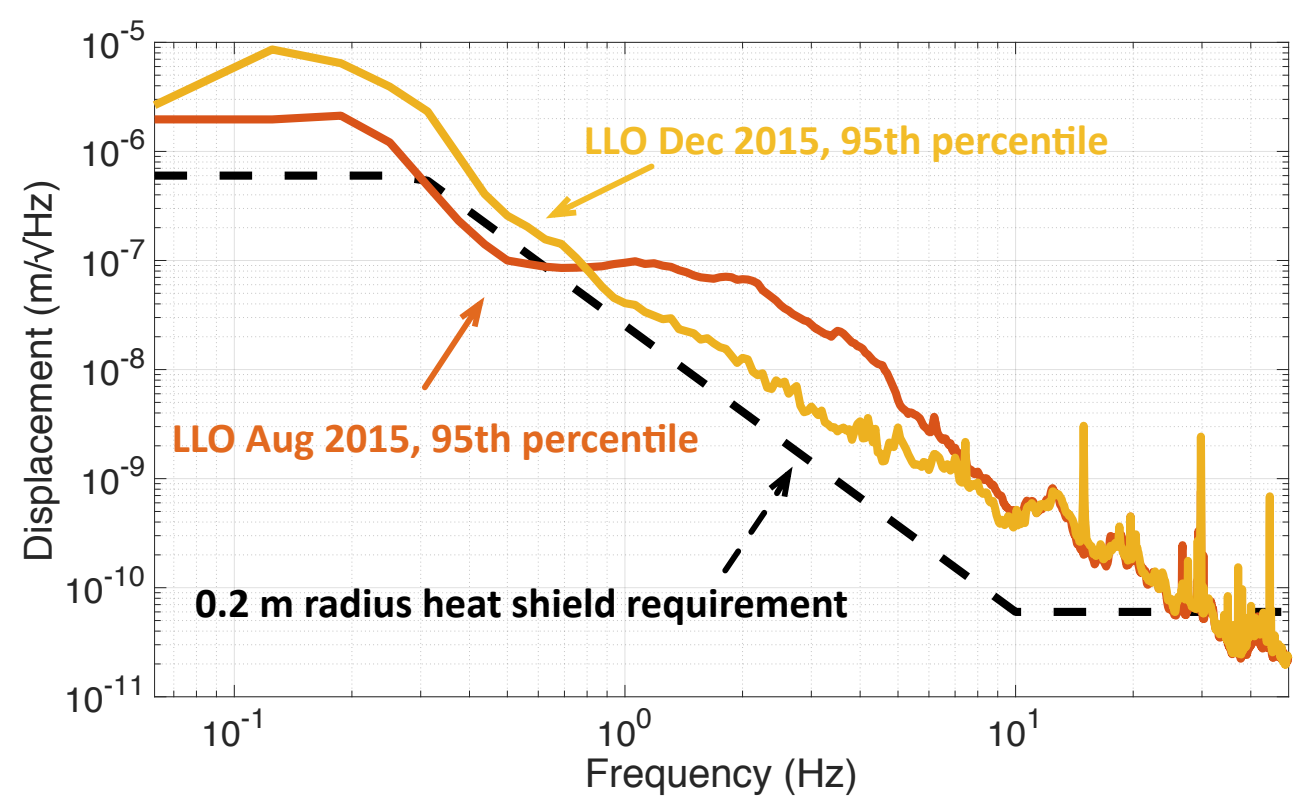

Figure 3. Measured Livingston Observatory (LLO) ground displacement compared to the inner shield displacement limit for a $0.2 \mathrm{~m}$ shield. The red curve is from August 2009, the yellow December 2009 [13]. These ground displacement curves represent the 95th percentile from that month, meaning the displacement was less $95 \%$ of the time.

2.3.2. Inner shield vibration isolation Because the ground displacements are often greater than the limit on the inner shield displacement, it will need vibration isolation. 
This isolation is achieved in two ways. First, it is suspended from the ground, which gives it isolation at frequencies above its suspension resonance at a few Hz. Second, the low frequency isolation is achieved using active feedback.

Fig. 4 presents a block diagram of this feedback. Relative displacement sensors are used between the inner shield and the nearby vibration isolation table. Feeding back these sensor signals forces the shield to have the same displacement as the vibration isolation table within the noise floor of the displacement sensors and the bandwidth of the feedback loop. These displacement sensors should have a noise floor no higher than the shields displacement limit. Existing Advanced LIGO displacement sensors have sufficient performance for a shield with an effective radius of at least $0.2 \mathrm{~m}$ [14]. The actuators react against the ground to minimize disturbances on the vibration isolation table. The vibration isolation table is itself well isolated with its own feedback loops by utilizing its low noise on-board inertial sensors $[15,16]$.

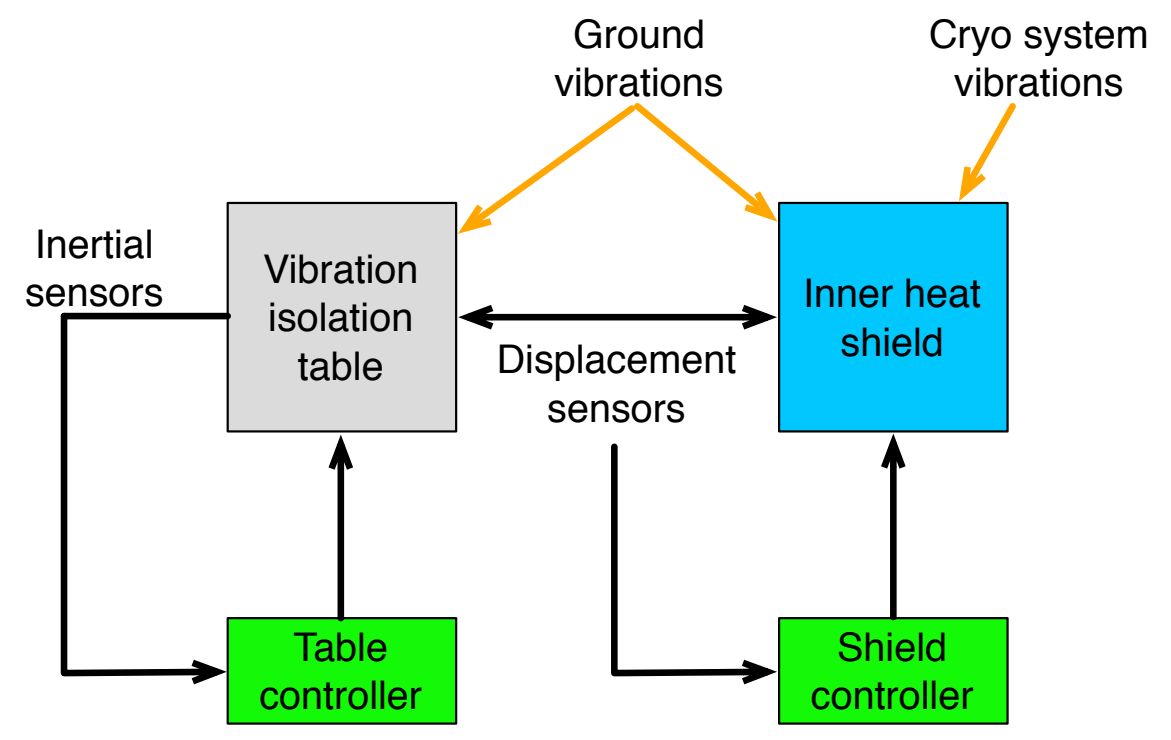

Figure 4. A block diagram of the feedback loop used to reduce the displacement of the inner shield. The vibration isolation table's displacement is well suppressed by feeding back its on-board inertial sensor signals. Displacement sensors then measure the relative displacement between the shield and the table. These signals are fed back to the inner shield to suppress its displacement.

\section{Experimental Setup at Stanford University}

To test this proposed low vibration cryogenic system, an experimental setup was put together at Stanford University. Fig. 5 shows a schematic of this setup and Fig. 6 shows a photograph. The configuration is very similar to that proposed in Fig. 2. Both have a two-stage active feedback vibration isolation table, a suspended silicon mirror, and a dual heat shield system. 


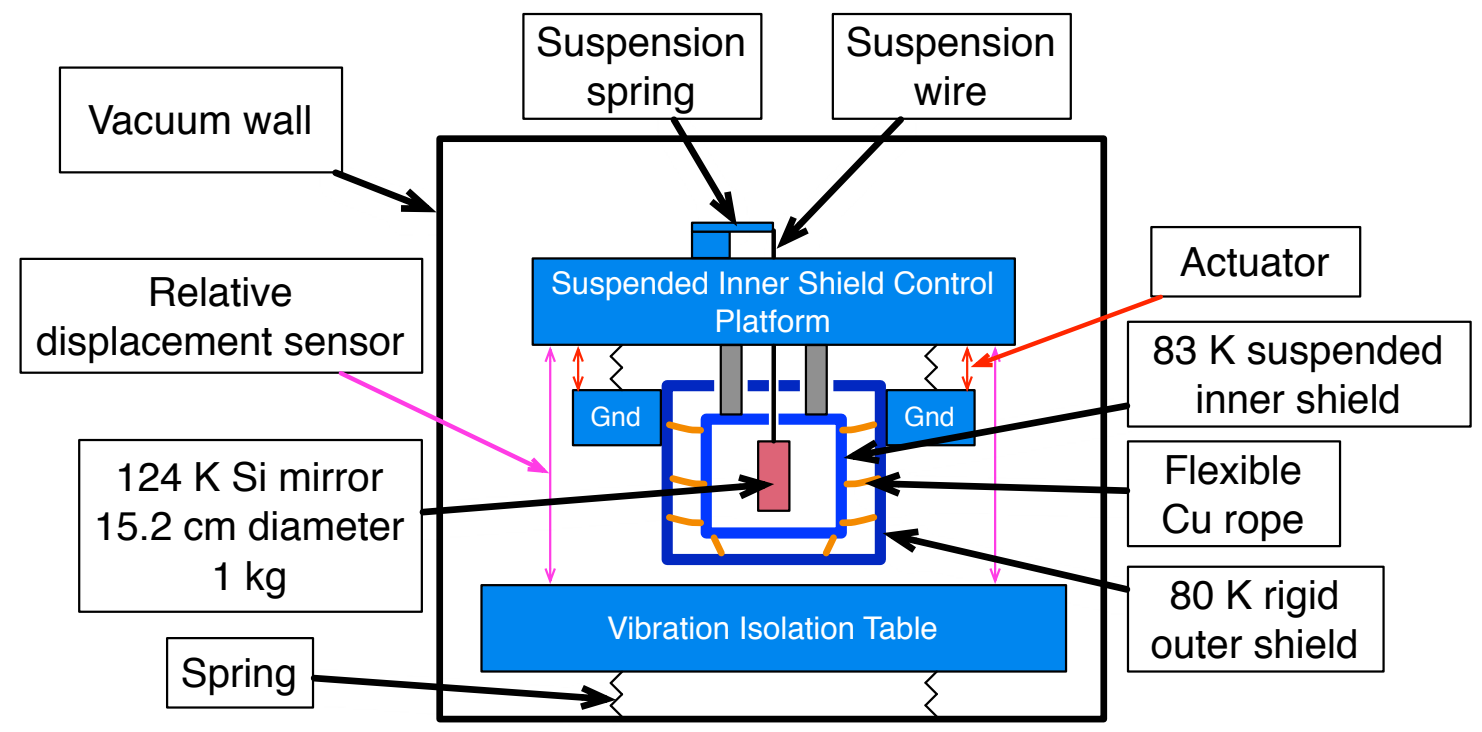

Figure 5. A schematic of the experimental setup at Stanford. This schematic is similar to that in Fig. 2, where in this case the vibration isolation table is underneath the mirror. The inner shield and the inner shield control platform are a single rigid unit suspended from the ground with $7 \mathrm{~Hz}$ to $10 \mathrm{~Hz}$ resonant frequencies. The inner shield's displacement is controlled through this platform utilizing displacement sensors referenced to the vibration isolation table and actuators reacting against the ground.

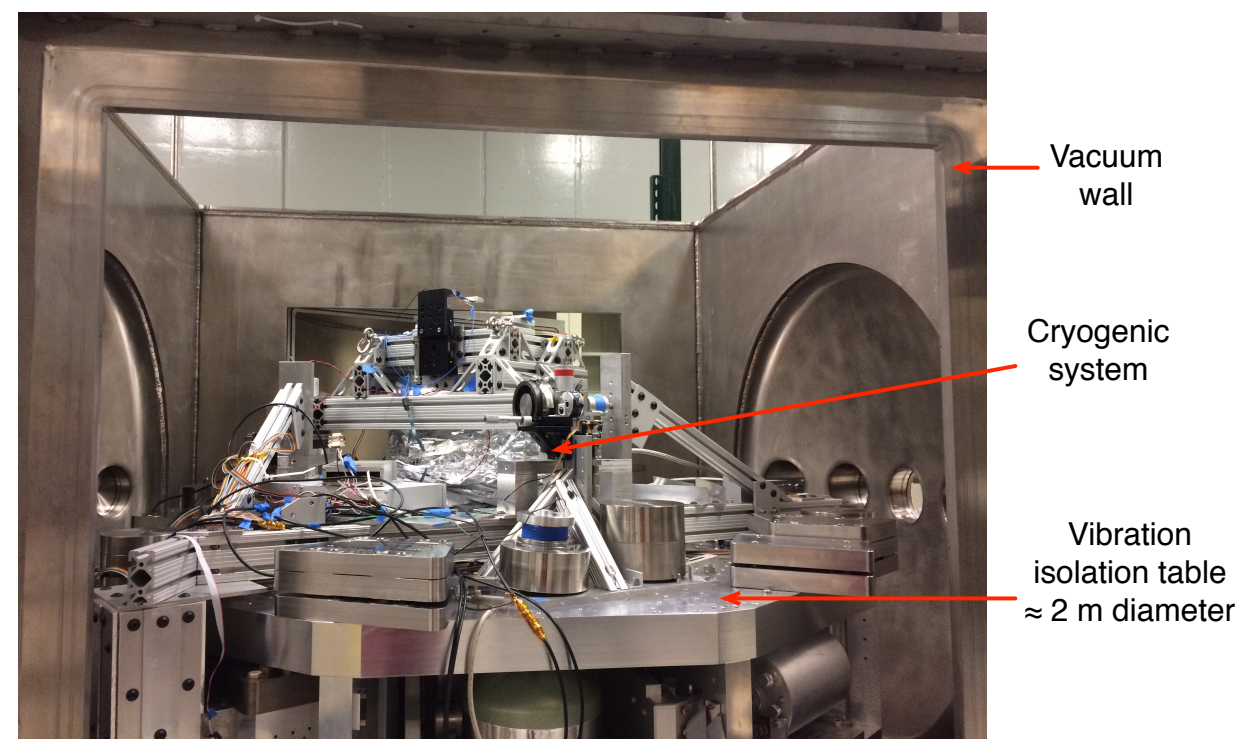

Figure 6. A photograph of the cryogenic setup inside the vacuum chamber at Stanford University. Here, the vibration isolation table is below the cryogenics. No part of the cryogenic system touches the vibration isolation table, but rather the system merely hangs above the table through a structure that is bolted to the ground. The roof of the chamber has been removed in order to install the cryogenic system over the vibration isolation table. 


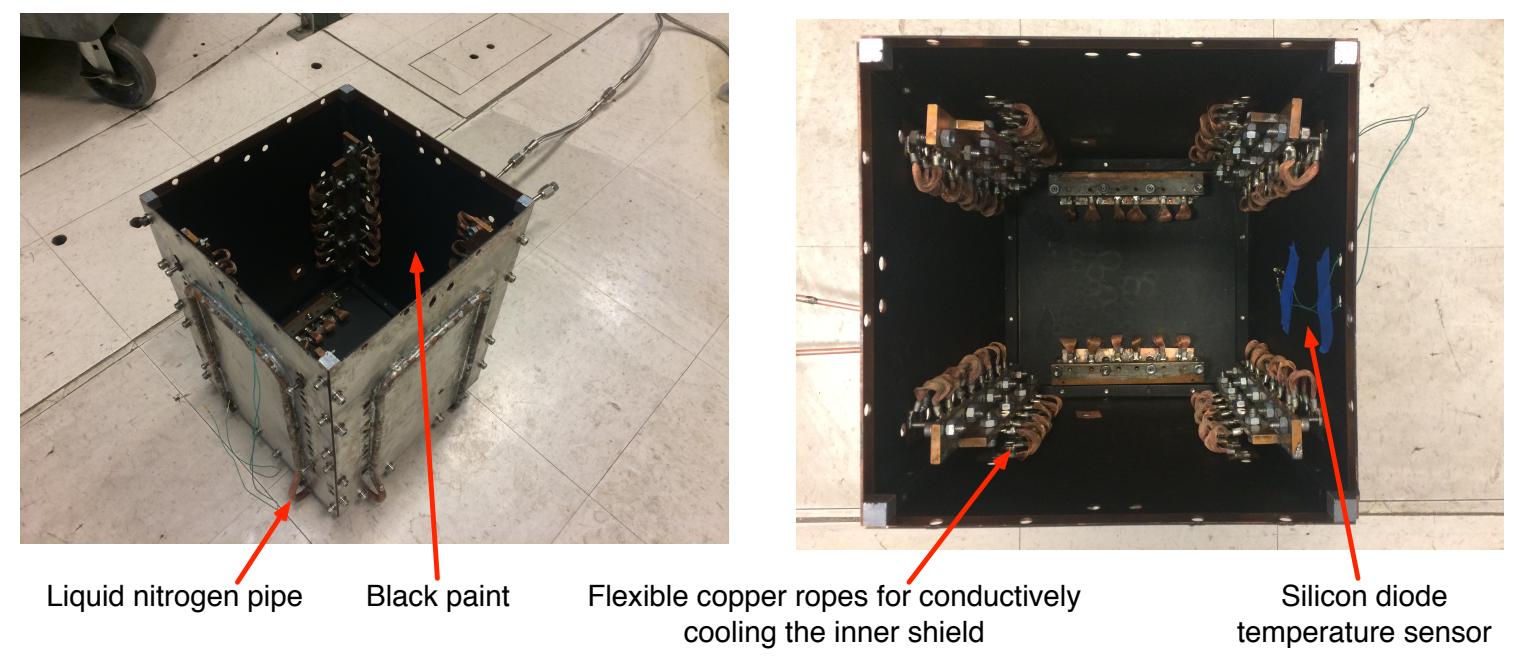

Figure 7. The outer shield, before integration with the inner shield. The outer shield is made of $1.6 \mathrm{~mm}(1 / 16 \mathrm{inch})$ copper sheet and is about $35 \mathrm{~cm}$ tall and $28 \mathrm{~cm}$ wide. Left image: a $1 \mathrm{~cm}(3 / 8$ inch) copper liquid nitrogen pipe soldered to the outside is used for cooling. The inside is painted black to improve the heat transfer from the inner shield. Right image: inside the shield, flexible copper ropes are used to conductively cool the inner shield. These ropes are $4.5 \mathrm{~cm}$ long, and each are made of 430 strands of 36 AWG copper wire $(0.127 \mathrm{~mm}$ diameter $)$. There are 68 total ropes. The cold end of the rope is soldered to the outer shield and the warm end is attached to the inner shield with bolts. The shield's temperature is monitored with a Lake Shore DT-670 silicon diode temperature sensor, rated to $\pm 32 \mathrm{mK}$.

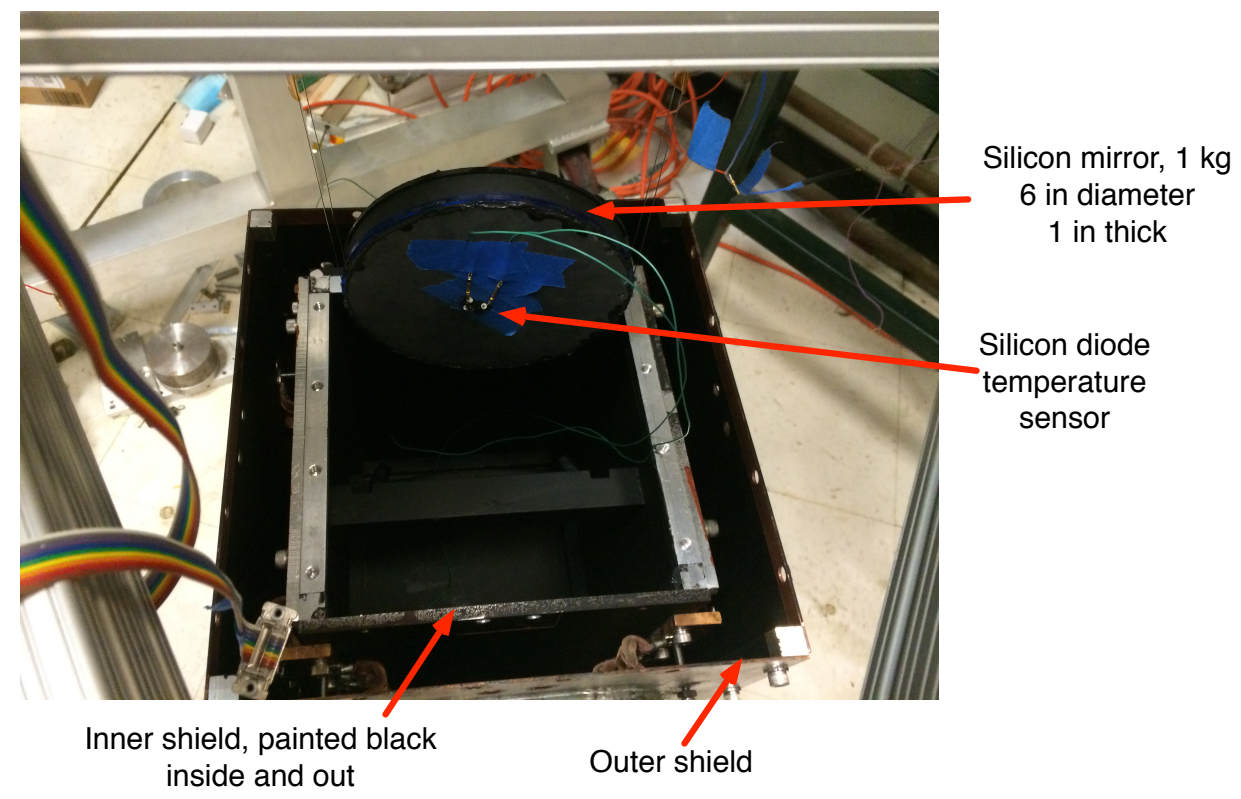

Figure 8. A photograph of the inner shield inside the outer shield while the silicon mirror is being installed. The inner shield is painted black on both the inside and outside surfaces. The mirror is also painted black on the cylindrical barrel. A Lake Shore DT-670 silicon diode temperature sensor is visible on the mirror. Here the two shields are temporarily bolted together for assembly and installation. Normally they are only connected by the flexible copper ropes. 


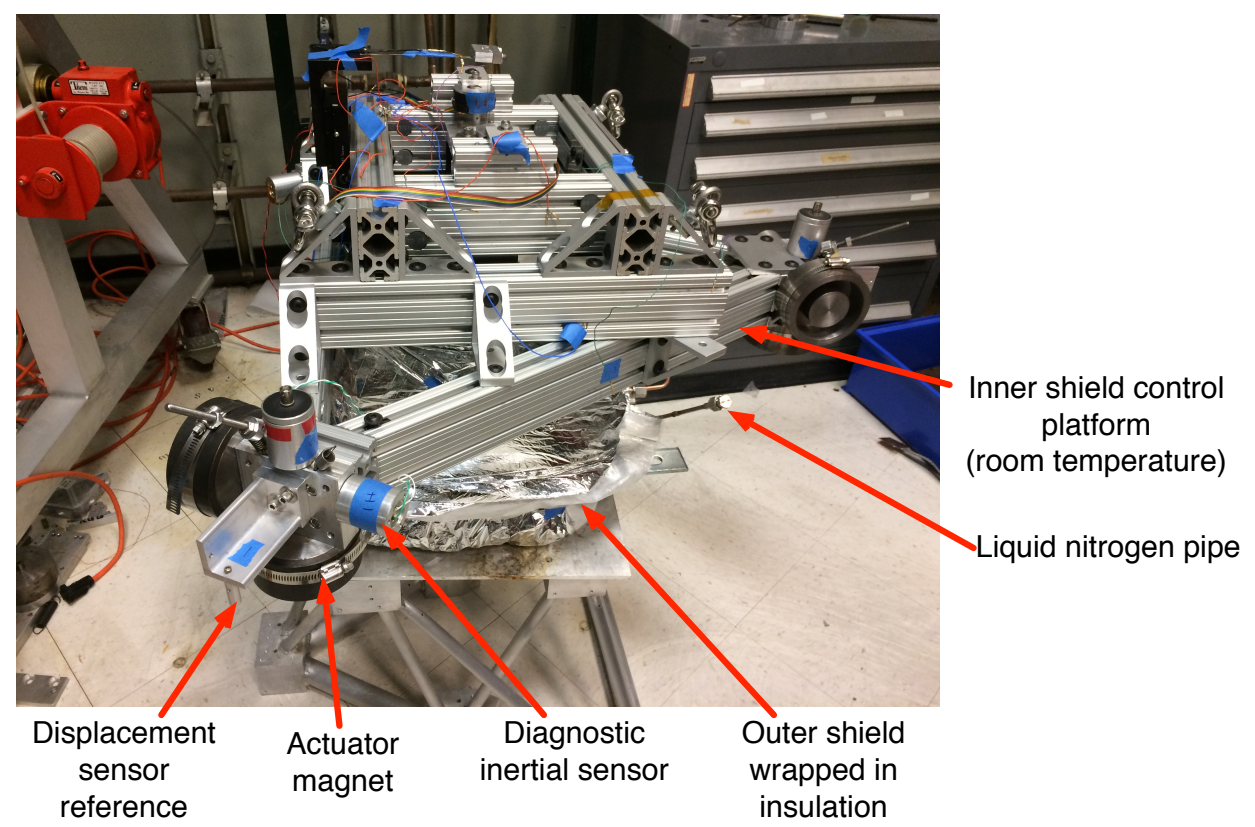

Figure 9. A photograph of the assembled cryogenic system before installation into the vacuum chamber. The inner shield is rigidly connected to an $80 / 20$ structure, the inner shield control platform, which remains warm. The sensors and actuators utilized for the inner shield's feedback control are mounted to this platform.

The setup at Stanford does have some differences, in part to simplify the experiment and reduce its scale, and in part due to the differences between the facilities at Stanford and the LIGO sites. First, the vibration isolation table sits underneath the mirror rather than above and faces up rather than down. The reason for this is historical, but the table functions in a nearly identical manner. Second, the cryogenic system is smaller than it would be at the LIGO sites. The mirror is $1 \mathrm{~kg}$ and $15.2 \mathrm{~cm}$ (6 inches) in diameter rather than between $150 \mathrm{~kg}$ and $200 \mathrm{~kg}$ and $44 \mathrm{~cm}$ in diameter. The inner shield is designed to handle heat loads of $10 \mathrm{~W}$ to $15 \mathrm{~W}$ rather than $100 \mathrm{~W}$. This smaller size reduces the cost and cycle time of this experiment. Third, the mirror hangs as a single stage suspension rather than a four stage suspension. This also reduces the cost and complexity of the experiment, and keeps it to a size that fits in the smaller Stanford chamber. The complexity of the suspension is not important for this experiment since we are not testing the isolation of the mirror itself, but rather that of the inner shield and the isolation table. Fourth, the Stanford mirror has no heat load on it. Adding a heat load to simulate the expected $5 \mathrm{~W}$ from the laser is planned for future work. Fifth, there is no beam tube shield. The heat shields fully enclose the mirror at Stanford. A beam tube shield is also planned in future work. These differences do not prevent this experiment from exploring the possibility of simultaneously achieving a cold mirror with a sufficiently low vibration inner shield and a low vibration isolation table.

The heat shields, mirror, actuators, and sensors are pictured in Figs. 7 to 9. The outer shield is made of $1.6 \mathrm{~mm}(1 / 16$ inch) copper sheet, and is about $35 \mathrm{~cm}$ tall and 28 
$\mathrm{cm}$ wide. It is cooled by a $1 \mathrm{~cm}(3 / 8$ inch) liquid nitrogen copper pipe soldered directly to its outer surface. Copper was chosen for its high thermal conductivity and for the ease of soldering copper pipe.

The inner shield is made of $0.635 \mathrm{~cm}(1 / 4 \mathrm{inch})$ aluminum 1100 plate. Here, this nearly pure aluminum alloy was chosen over copper primarily because the ratio of its Young's Modulus to its density is higher. This maximizes the shield's internal resonant frequencies, which is important for good feedback control. Additionally, this alloy's thermal conductivity, being half that of copper's, is still high. Also, since it is lighter than copper, a thicker wall can be used to get similar conductivity with about the same weight. The aluminum alloy also costs less than copper.

The inner shield is primarily cooled conductively to the outer shield through flexible copper ropes between the shields. There are 68 of these ropes, which are each $4.5 \mathrm{~cm}$ long and made of 430 strands of 36 AWG copper wire $(0.127 \mathrm{~mm}$ diameter). The ropes are soldered to the outer shield on one end and bolted to the inner shield on the other. While bolted joints are known to have finite thermal conductivity, they were chosen to simplify the assembly and maintenance of the system. To minimize their resistance, the joints were given as much surface area as possible by soldering the ropes to large flat copper bars, which are then bolted to the inner shield. To aid the inner shield's heat transfer to/from all other neighboring surfaces, LORD Corporation Z306 Flat Black [17] was used on the inside surface of the outer shield, both inside and outside surfaces of the inner shield, and the cylindrical barrel of the mirror.

It is estimated that the steady state heat load on the inner shield is a maximum of $10 \mathrm{~W}$, mainly due to the conductive heat transfer from its physical connection to the outside world. It is expected there will also be a radiative heat leak through the top of the outer shield. The top is not cooled directly with liquid nitrogen pipes, which allows for the mirror to be installed through the top. The size of this leak is minimized by using multi-layer insulation on the top of the shield. The 68 copper ropes together are predicted to have a thermal conductance of $3.7 \mathrm{~W} / \mathrm{K}$. Thus, if the temperature difference between the shields is $3 \mathrm{~K}$, the ropes will transfer $11 \mathrm{~W}$, assuming no extra thermal resistance in the bolted joints are anywhere else. $4 \mathrm{~K}$ will transfer $15 \mathrm{~W}$.

The feedback control of the inner shield is set up the same way as shown in the block diagram in Fig. 4. To implement this feedback, the shield rigidly hangs from the inner shield control platform sketched in Fig. 5. The platform is made of 80/20 and is visible in Fig. 9. The inner shield and control platform are suspended as a single unit from the ground with resonant frequencies between $7 \mathrm{~Hz}$ and $10 \mathrm{~Hz}$. The control platform remains warm and contains the inner shield's sensors and actuators. The actuators are electromagnetic utilizing coil-magnet pairs. The magnets are connected to the control platform while the coils (not visible) are mounted to the ground, which serves as the reaction platform. The displacement sensors are mounted to the isolation table, and therefore not shown in Fig. 9. However, one of their reference points, a small cylindrical stick, is visible. The sensors are shadow sensors [14], and this stick creates the shadow that generates the displacement signal. Also visible are inertial sensors, 
HS1 geophones. These are not used in the feedback, but serve to help characterize the shield's displacement with an out-of-loop sensor and for diagnostic tests of the system.

\section{Results and Analysis}

\subsection{Target results}

The experimental setup at Stanford was judged on both its vibration isolation and thermal performance. The primary thermal goal is to get the inner shield to $83 \mathrm{~K}$. The secondary goal is to cool the mirror to $124 \mathrm{~K}$. The authors assume for this work that if the shield can be brought to the right temperature, then the mirror can also be brought to the right temperature. This assumption will be explored more deeply in future work.

The vibration goal of the inner shield has to be modified somewhat, given the differences in the seismic environments between Stanford University and the LIGO sites. Consequently, rather than trying to achieve a particular displacement spectrum, the goal is to achieve a particular amount of vibration suppression. The suppression goal for this experiment is given by the blue curve in Fig. 10. This suppression is the ratio between the displacement limit curve for a $0.2 \mathrm{~m}$ shield and the maximum of the ground motion at Livingston shown in Fig. 3. If the experimental setup was used at Livingston, it would therefore be expected to achieve the displacement limit curve. Additionally, to minimize the impact on the mirror's isolation, the vibration of the isolation table must not be increased by the shield's feedback control or by the liquid nitrogen flow.

\subsection{Achieved results}

The red curve in Fig. 10 shows the vibration suppression achieved using the feedback loop gain design in Fig. 11. At all frequencies, the measured suppression is no higher than the required suppression. Thus, the inner shield achieves the required vibration performance. Fig. 12 shows the vibration spectrum of the isolation table with the cryogenic system in 3 configurations: feedback and liquid nitrogen flow both off, feedback off with the nitrogen flow on, and with both on. In all three cases, the vibration is virtually the same, indicating that the cryogenic system is not impacting the vibration isolation of the mirror.

Fig. 13 shows the thermal performance of the two shields and the mirror. The outer and inner shields reach $79.5 \mathrm{~K}$ and $83.5 \mathrm{~K}$ respectively. After 660, minutes the liquid nitrogen source ran out and the system began to warm up again. After the nitrogen ran out, the mirror continued cooling down until it reached $121 \mathrm{~K}$ before warming up again. Thus, all cold components reached their desired temperatures.

Calculated from the inner shield's cool-down curve and its estimated heat capacity, the shield's heat leak is about $12 \mathrm{~W}$, slightly higher than the predicted $10 \mathrm{~W}$. Similarly, the thermal conductivity of the copper ropes is $80 \%$ of the design value. At this point, it is unknown how much of the extra resistance is from the bolted joint. Future work 
will explore the bolted joint resistance and how much the mirror's cool-down rate can be improved.

The temperature of the suspension spring the mirror hangs from was also investigated. This spring remains outside the cryogenic system and is intended to remain at room temperature. In Advanced LIGO, which nominally operates entirely at room temperature, changes in the springs of $\pm 1 \mathrm{~K}$ are large enough to misalign the interferometer. The change in temperature changes the spring's Young's Modulus and thus the spring's stiffness, resulting in height and angle changes in the mirror's position. Fig. 14 shows the temperature response of the spring in the Stanford experiment from the cryogenics. In this case, the spring is relatively close to the outer shield, about $15 \mathrm{~cm}$ away. It's temperature drops $29 \mathrm{~K}$ throughout the experiment. The heat transfer out of the spring is through a combination of radiation and conduction. It could be improved somewhat by thermally isolating the spring more robustly from the shield. However, it seems likely that some eventual temperature control of the suspension springs will be desirable.

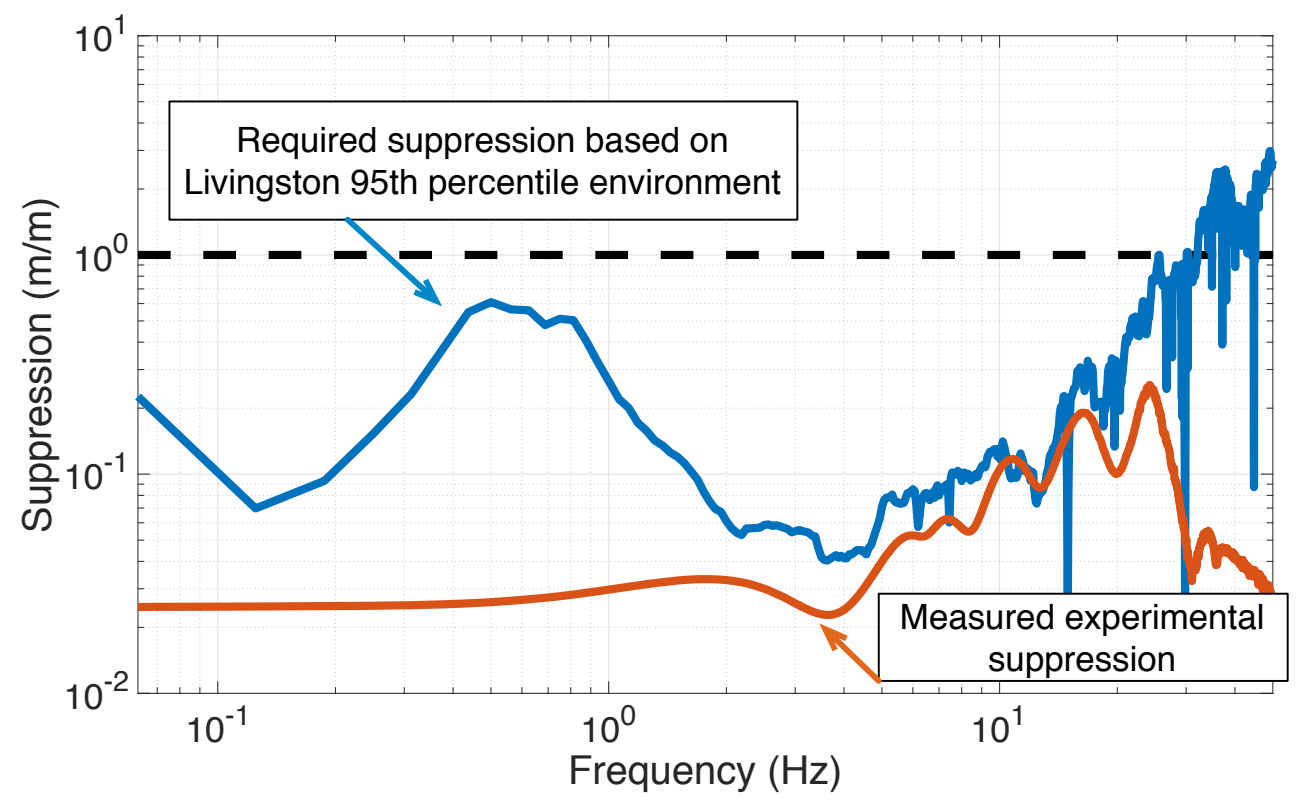

Figure 10. Ground displacement suppression. Blue: the required suppression of the Livingston ground displacement for an inner shield to meet the displacement requirement in Fig. 3. Red: the measured suppression achieved with the Stanford experiment using active feedback. 

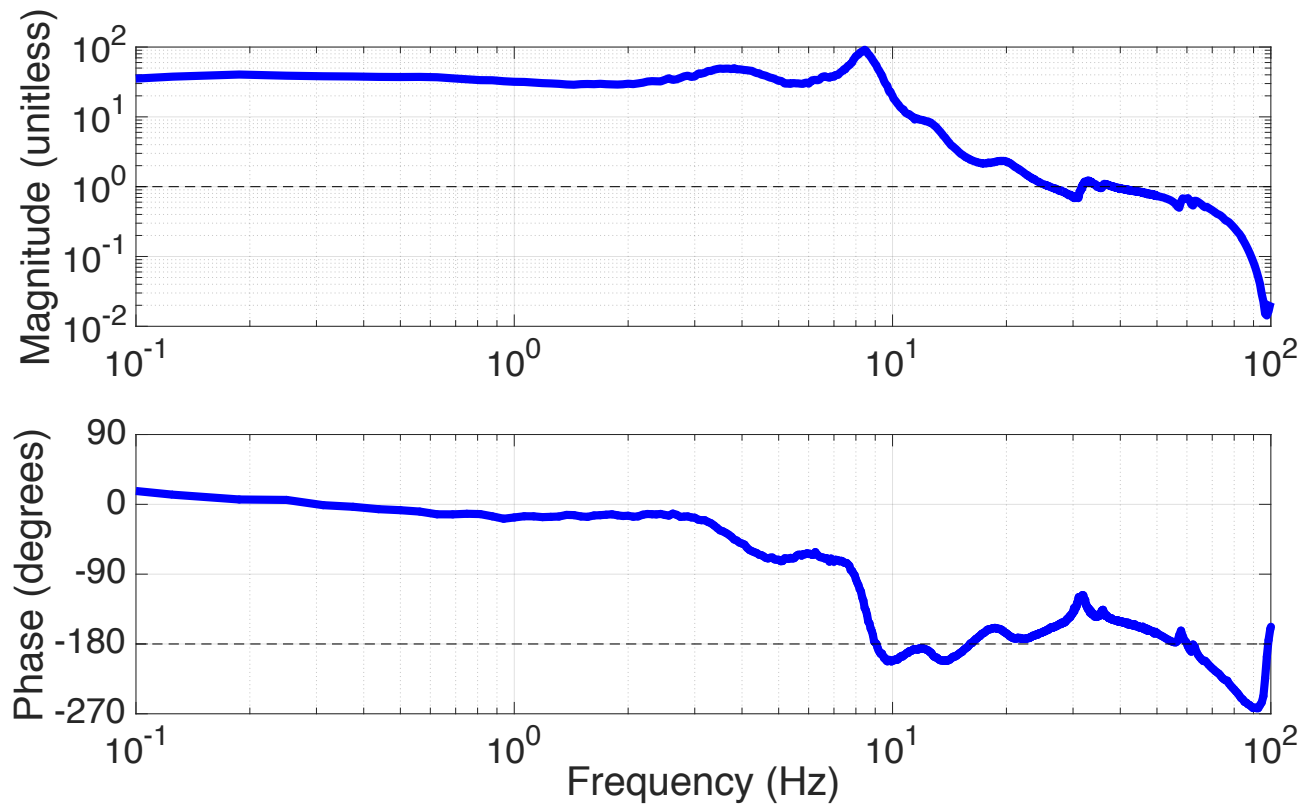

Figure 11. The loop gain of the active feedback design used on the Stanford experiment to achieve the suppression shown in Fig. 10 in red.

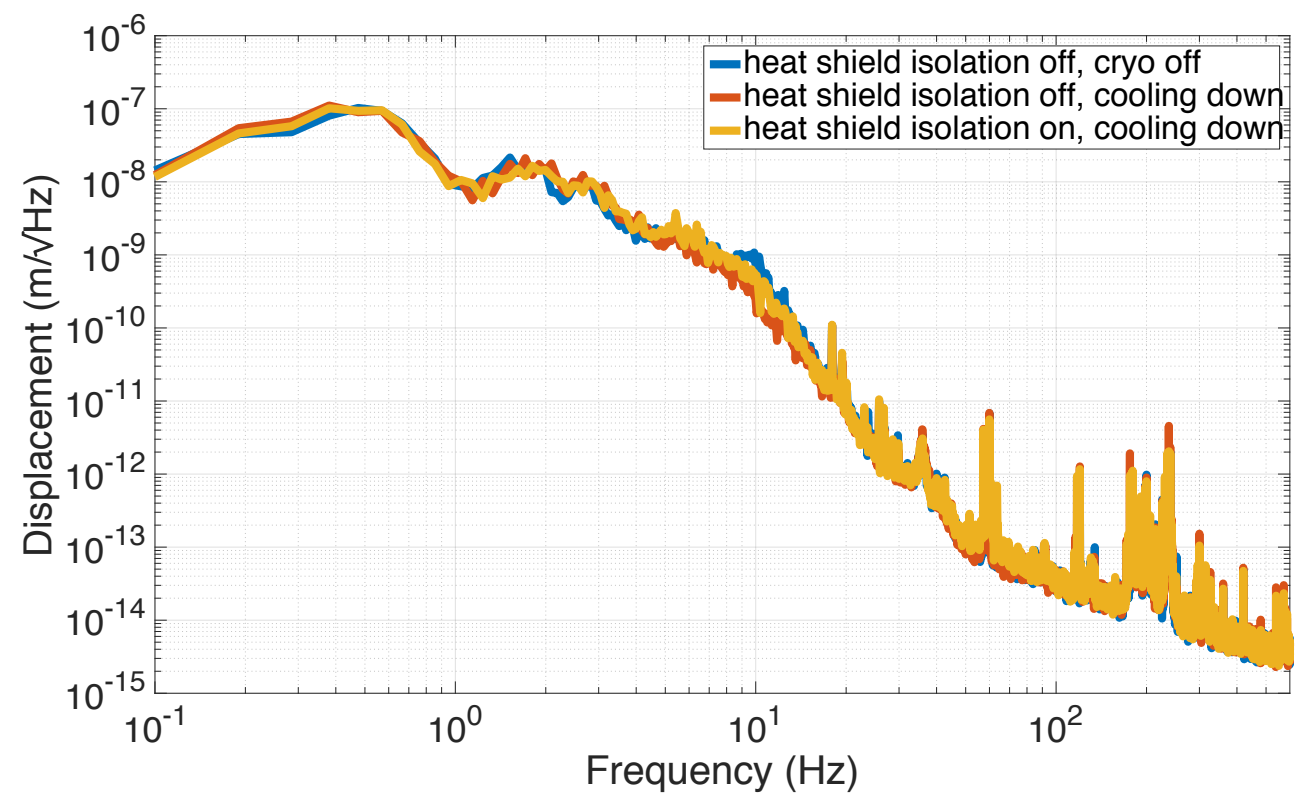

Figure 12. The displacement of the vibration isolation table used in the Stanford experiment. The baseline displacement, blue, is largely unaffected by the introduction of a liquid nitrogen flow, red, and the active feedback on the inner shield, yellow. 


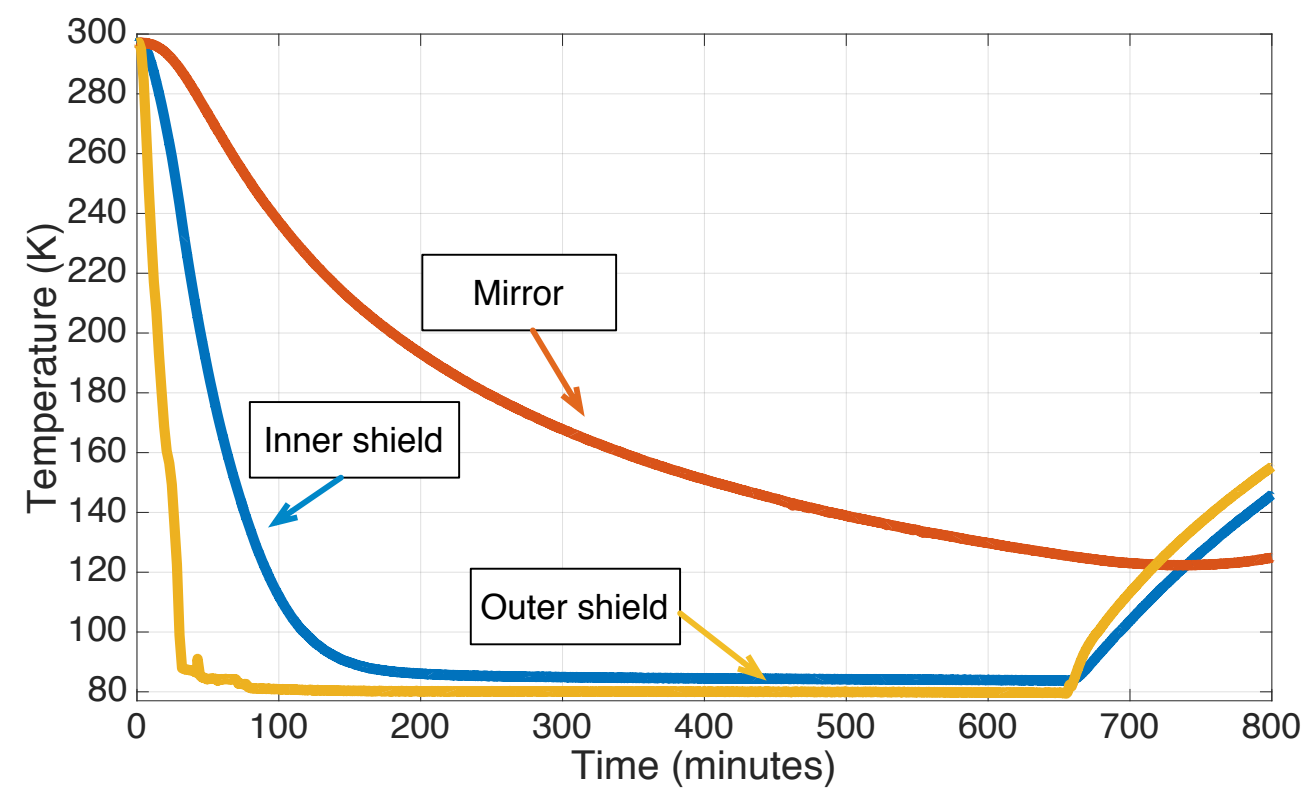

Figure 13. The thermal response of the two shields and the mirror to the liquid nitrogen flowing through the outer shield pipes. The nitrogen flow is turned on at minute 0 and runs out at minute 660 .

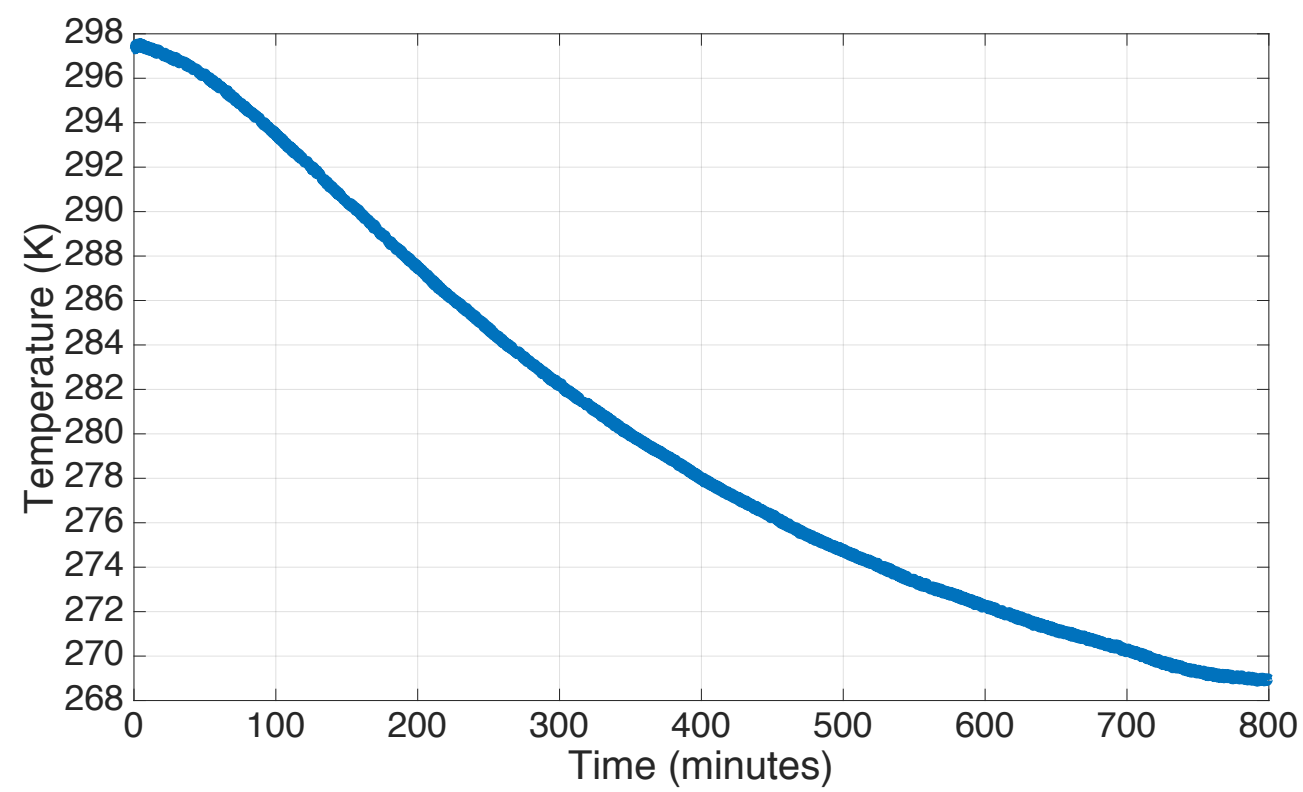

Figure 14. The temperature evolution of the cantilever spring supporting the mirror. This spring is outside the heat shields and intended to stay at room temperature with a precision of $\pm 1 \mathrm{~K}$. The time axis is coincident with Fig. 13.

\section{Conclusions}

The results from the Stanford cryogenic mirror experiment indicate that it is feasible to cool a mirror to $124 \mathrm{~K}$ with a negligible increase in the displacement noise spectrum 
of a future LIGO Voyager style gravitational wave interferometer. This cooling has the potential to contribute to a factor of 3 reduction of noise in LIGO Voyager compared to existing Advanced LIGO observatories.

The design of the experiment at Stanford is expected to be scalable in size to a LIGO Voyager facility. However, design work will be needed to ensure similar performance with the higher heat loads and larger geometries.

Additional work is also still required before the technology is sufficiently mature. The cool-down rate of the mirrors needs to be improved in order avoid waiting weeks for the interferometer to be operational each time it is thermally cycled. A heat shield for the beam tube needs to be developed so that an access hole for the laser can be built into the mirror's heat shields without generating a large heat leak. The mirror's springs will need their own temperature control system to avoid misaligning the interferometer with large temperature drifts. While not expected to be an issue, a possible source of noise from the rubbing of the wire strands in the copper ropes needs to be ruled out. Finally, a high emissivity, low mechanical loss coating for the mirror must be identified so that its heat can be radiated away without compromising its thermal noise performance.

\section{Acknowledgments}

This work was supported by funding from the National Science Foundation under cooperative agreements PHY-1404430. The authors would like to thank Rainer Weiss and Warren Johnson for many useful discussions, without which this work would not have been possible. The authors would like to acknowledge the contributions of Professors Bob Byer to this work.

LIGO was constructed by the California Institute of Technology and Massachusetts Institute of Technology with funding from the National Science Foundation and operates under cooperative agreement PHY-0757058. This paper has LIGO Document Number LIGO-P1600301.

\section{References}

[1] B. P. Abbott, R. Abbott et al., "Observation of gravitational waves from a binary black hole merger," Phys. Rev. Lett., vol. 116, p. 061102, Feb 2016. [Online]. Available: http://link.aps.org/doi/10.1103/PhysRevLett.116.061102

[2] —, "GW151226: Observation of gravitational waves from a 22-solar-mass binary black hole coalescence," Phys. Rev. Lett., vol. 116, p. 241103, Jun 2016. [Online]. Available: http://link.aps.org/doi/10.1103/PhysRevLett.116.241103

[3] The LIGO Scientific Collaboration et al., "Advanced LIGO," Classical and Quantum Gravity, vol. 32, no. 7, p. 074001, 2015. [Online]. Available: http://stacks.iop.org/0264-9381/32/i=7/a $=074001$

[4] B. P. Abbott, R. Abbott et al., "GW150914: The Advanced LIGO detectors in the era of first discoveries," Phys. Rev. Lett., vol. 116, p. 131103, Mar 2016. [Online]. Available: http://link.aps.org/doi/10.1103/PhysRevLett.116.131103

[5] A. Einstein, "Approximative Integration of the Field Equations of Gravitation," Sitzungsber. Preuss. Akad. Wiss. Berlin (Math. Phys.), vol. 1916, pp. 688-696, 1916. 
[6] P. B. Karlmann, K. J. Klein, P. G. Halverson, R. D. Peters, M. B. Levine, D. Van Buren, and M. J. Dudik, "Linear thermal expansion measurements of single crystal silicon for validation of interferometer based cryogenic dilatometer," AIP Conference Proceedings, vol. 824, no. 1, pp. 35-42, 2006. [Online]. Available: http://scitation.aip.org/content/aip/proceeding/aipcp/10.1063/1.2192331

[7] T. Akutsu and K. collaboration, "Large-scale cryogenic gravitational-wave telescope in Japan: KAGRA," Journal of Physics: Conference Series, vol. 610, no. 1, p. 012016, 2015. [Online]. Available: http://stacks.iop.org/1742-6596/610/i=1/a=012016

[8] M. Abernathy, F. Acernese et al., "Einstein gravitational wave telescope conceptual design study," 2011. [Online]. Available: https://tds.ego-gw.it/itf/tds/index.php?callContent $=2 \&$ callCode $=$ 8709

[9] D. Madden-Fong, B. Shapiro, and B. Lantz, "Radiation Transfer at the Face of a LIGO III Test Mass," LIGO, Internal Technical Document T1300556, 2013. [Online]. Available: https://dcc.ligo.org/LIGO-T1300556/public

[10] Y. Sakakibara, T. Akutsu, D. Chen, A. Khalaidovski, N. Kimura, S. Koike, T. Kume, K. Kuroda, T. Suzuki, C. Tokoku, and K. Yamamoto, "Progress on the cryogenic system for the KAGRA cryogenic interferometric gravitational wave telescope," Classical and Quantum Gravity, vol. 31, no. 22, p. 224003, Nov. 2014. [Online]. Available: http://adsabs.harvard.edu/abs/2014CQGra..31v4003S

[11] Y. Sakakibara, N. Kimura, T. Akutsu, T. Suzuki, and K. Kuroda, "Performance test of pipe-shaped radiation shields for cryogenic interferometric gravitational wave detectors," Classical and Quantum Gravity, vol. 32, no. 15, p. 155011, 2015. [Online]. Available: http://stacks.iop.org/0264-9381/32/i=15/a=155011

[12] S. Zeidler, T. Akutsu, Y. Torii, Y. Aso, F. Erasmo, P. Arellano, J. Katayama, K. Yamamoto, Y. Sakakibara, and R. Flaminio, "Optical Measurements and Calculations for KAGRA - Performing Stray-Light Control," 2015. [Online]. Available: http: //gwdoc.icrr.u-tokyo.ac.jp/DocDB/0034/G1503403/005/JPS-presentation_SimonZeidler.pdf

[13] J. Harms, "Seismic spectral analysis; LHO/LLO LVEA 2009/2010," LIGO, Internal Technical Document T1500224, 2015. [Online]. Available: https://dcc.ligo.org/LIGO-T1500224/public

[14] L. Carbone, S. M. Aston, R. M. Cutler, A. Freise, J. Greenhalgh, J. Heefner, D. Hoyland, N. A. Lockerbie, D. Lodhia, N. A. Robertson, C. C. Speake, K. A. Strain, and A. Vecchio, "Sensors and actuators for the Advanced LIGO mirror suspensions," Classical and Quantum Gravity, vol. 29, no. 11, p. 115005, Jun. 2012. [Online]. Available: http://adsabs.harvard.edu/abs/2012CQGra..29k5005C

[15] F. Matichard, B. Lantz et al., "Advanced LIGO two-stage twelve-axis vibration isolation and positioning platform. part 1: Design and production overview," Precision Engineering, vol. 40, pp. $273-286$, 2015. [Online]. Available: http://www.sciencedirect.com/science/article/pii/S0141635914001561

[16] _ - "Advanced LIGO two-stage twelve-axis vibration isolation and positioning platform. part 2: Experimental investigation and tests results," Precision Engineering, vol. 40, pp. 287 - 297, 2015. [Online]. Available: http://www.sciencedirect.com/science/article/pii/S0141635914002098

[17] M. A. Quijada, J. G. Hagopian, S. Getty, R. E. Kinzer, Jr., and E. J. Wollack, "Hemispherical reflectance and emittance properties of carbon nanotubes coatings at infrared wavelengths," pp. 815 002-815 002-11, 2011. [Online]. Available: http://dx.doi.org/10.1117/12.894601 